«Du média à la médiation : les trente ans de la pensée intermédiale et la résistance théâtrale »

\author{
Jean-Marc Larrue \\ dans Théâtre et intermédialité, Villeneuve-d'Ascq, Presses universitaires du Septentrion, coll. «Arts du spectacle. \\ Images et sons », 2015, p. 27-56.
}

Pour citer ce chapitre :

LARRUE, Jean-Marc, «Du média à la médiation : les trente ans de la pensée intermédiale et la résistance théâtrale », dans Jean-Marc Larrue (dir.), Théâtre et intermédialité, Villeneuve-d'Ascq, Presses universitaires du Septentrion, coll. «Arts du spectacle. Images et sons », 2015, p. 27-56. 


\section{Du média à la médiation : les trente ans de la pensée intermédiale et la résistance théâtrale}

Jean-Marc Larrue

Si les études intermédiales, qui sont nées dans le sillage de la « révolution numérique », ont à peine trente ans, les processus qu'elles contribuent à mettre au jour remontent bien au-delà de cette dernière vague technologique majeure, comme l'a clairement illustré Remediation. Understanding New Media, l'ouvrageclé que Jay David Bolter et Richard Grusin publiaient (en version imprimée) en $2000^{1}$. Selon certains chercheurs, tel Peter Boenisch, les processus intermédiaux sont présents dès l'invention de l'alphabet ${ }^{2}$ et les théoriciens de l'interartialité (Walter Moser ${ }^{4}$, Claus Clüver ${ }^{5}$ ) voient dans les relations complexes et constantes entre les pratiques artistiques depuis l'antiquité des modèles probants de dynamique intermédiale. Du point de vue des premiers intermédialistes, en effet, les arts sont des médias, ce sont même les plus anciens médias.

1-Jay David Bolter (Massachusetts), MIT Press, 2000 [1999], 295 p.
. Understanding New Media, Cambridge

dans la Grèce antique de Derrick de Kerckhove pour lequel l’origine du théâtre occidental, tique deux ou antique, est directement attribuable au développement de l'alphabet phonéparticulier Derrick de Kes avant les premières Dionysies (vers le VIII ${ }^{c}$ siècle av. J-C) Voir en iss. 29, 1980 p. 23-25. Kerckhove, « A Theory of Greek Tragedy 》, SubStance, vol 4 $\pi^{\circ} 9$ Darroch (infra, p. 251) aborde cette quesion dans le texte Qu consacre au son et à la voix.

- Voir Walter Moser $<$ L' comme l'intermédialité restreinte aux seules pratiques artistiques. Ftoger et Jürgen $\mathrm{E}$. Mülinterartialité : pour une archéologie de l'intermédialité », in Marion coll. « Film und Med.

- Voir Claus Clïver, skander, Jor th intermediality, Luhn et Heidrun Führer (dir.), Changing Borders. Contemporary Positions

- 
Quant aux travaux menés en archéologie des médias depuis une trentaine d'années sur cette autre révolution qu'a provoquée l'avènement de l'électricité, ils montrent bien, par les similitudes frappantes qu'on observe dans le développement des technologies électriques et la formation des médias électriques, d'une part, et dans celui des technologies et médias numériques, d'autre part, que les mêmes phénomènes sont à l'ouvré à cent ans d'écart.

Marquée jusqu'au milieu des années 2000 , soit pendant près de vingt ans, par une conception assez traditionnelle du média, l'intermédialité est née d'un constat paradoxal : les médias, qui interagissent continuellement les uns avec les autres, sont, eux-mêmes, le produit de ces interactions. Mais depuis une dizaine d'années, le concept même de média est remis en question, et ce à un point tel qu'on se demande si une telle chose existe, a existé. On peut ainsi dégager deux qu'on se demande si une telle chose existe, a existé. On peut án l'intermédialité. La période médiapériodes de l'histire re construit sur une idée traditique et la période postmédiatique. La première se construit sur une idée traditionnelle du média, la seconde s'en dégage. La transition de l'une à l'autre illustre l'émancipation, laborieuse mais réussie, de l'intermédialité du régime de pensée poststructuraliste d'où elle a émergé.

\section{La période médiatique de l'intermédialité}

Rappel bistorique : média et intermédialité

L'intermédialité, telle que nous la comprenons, est à la fois un objet, une dynamique et une approche. Comme objet, elle concerne les relations complexes, foisonnantes, polymorphes, multidirectionnelles entre les médias ${ }^{7}$. Comme dynamique, l'intermédialité est ce qui permet l'évolution et la création de médias, elle est aussi ce qui produit des résidus médiatiques. Il découle de cela la nécessité d'une approche originale susceptible de mieux comprendre cet objet et cette dynamique.

Dès son origine, ainsi que nous allons le voir, la réflexion intermédiale a tenu compte de la place des usagers dans la réalité médiatique, ce qui a amené les

6.- Dès 1988, Carolyn Marvin publiait un ouvrage marquant sur la question, soulignant, elle

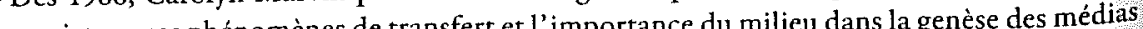
aussi, tous ces phenomes électriques (voir When Old Technologies Were New. Thinking About Electric Communication in the Late Nineteenth Century, New York, Oxford University Press, 1988, p. 269). Les ouvrages subséquents de Lisa Gitelman (Always Already New. Media, History and the Data of Culture, Cambridge [Massachusets]/Londres, MIT Press, 2006, 205 p.), de Siegfried Zielinski (Deep Time of the Media. Toward an Archeology of Hearing and Seing by Technical Means, Cambridge [Masto

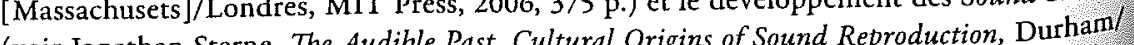
(voir Jonathan Sterne, The Audible Past. Cultural Origins of Sound Reproduction, Durhamble Londres, Duke University Press, 2003, 450 p.) participent du même mouvement d'ensemble
et témoignent autant de sa vitalité que de son éclectisme.

7.- Nous conservons ce terme tout en insistant sur son caractère problématique. intermédialistes à accorder une attention soutenue aux milieux (sociaux) d'où émergent les médias et sur lesquels ils agissent. Les socialités occupent donc une position centrale chez les intermédialistes, y compris chez les premiers intermédialistes qu'on a souvent accusés à tort d'être technocentristes. Les socialités liées aux médias ou créées par les médias - des premiers usagers du téléphone aux réseaux sociaux actuels - ne sont assimilables ni à des classes sociales ni à des communautés stables, d'où le recours au néologisme de «sociomédialités 》 pour définir ces socialités médiatiques en continuelle mutation.

Étymologiquement, le terme intermédialité renvoie à l'《 intermédia », qui recouvre, comme sa morphologie l'indique, ce qui se situe entre les médias. Le mot intermédia est d'abord apparu dans le champ des néo-avant-gardes au cours des années 1960. C'est au poète et compositeur Dick Higgins, cofondateur du mouvement d'art multidisciplinaire Fluxus, qu'on en attribue la paternitér. En multipliant les expérimentations de décloisonnement et de transfert entre disciplines, médias, techniques et technologies, Higgins et ses collègues de Fluxus produisaient effectivement des cuvres qu'on qualifierait aujourd'hui d'intermédiales, mais leur démarche reposait sur une conception somme toute classique du média qu'ils considéraient comme une entité discrète, entendons par là identifiable, isolable et relativement stable et autonome - à l'image des grands médias électriques (radio, télévision, cinéma) qui dominaient l'époque. Perçus comme des monades, les médias étaient définis en fonction de leur action sur le réel ou en fonction de leur structure et de leur mode de fonctionnement.

Friedrich Kittler, Frédéric Barbier et Catherine Bertho-Lavenir participent de la première tendance, ils définissent les médias par ce qu'ils font : pour Kittler, « un média archive des choses, transmet des choses ou traite des choses ${ }^{9} »$. Pour Barbier et Bertho-Lavenir, est média :

[t] out système de communication permettant à une société de remplir tout ou une partie des trois fonctions essentielles de la conservation, de la communication à distance des messages et des savoirs, et de la réactualisation des pratiques culturelles et politiques ${ }^{10}$.

8. - Rendu célèbre

Neusletter (volume son essai «Intermedia » publié en 1966 dans The Something Else est.org /20 volume 1, numéro 1). L'essai est disponible au $<$ http://www.withoutborders'ouvrage de JSEP_Newsletter..V1N1.pdf > (consultation le 13 juillet 2014). VoirdersQurage de Jacques Donguy et Charles Dreyfus, Dick Higgins, 1938-1998. Voir aussi Québec, Éditions Intervention, 1999, 20 p. Higgins préci Higgins, 1938-1998. Intermédia, sant les textes théoriques du poète, essayiste et critique que le concept lui est apparu en (1772-1843).

Friedrich Kitler, « media either store things, transmit things, or process things 》,

2013 [2012], p. 13. par Alexander R. Galloway, The Interface Effect, Cambridge, Polity,

Fridéric Basbi 13.

coll. «U $\gg$, Paris, Armand Corine Bertho-Lavenir, Histoire des médias. De Diderot à Internet, 
Ces définitions ne mentionnent ni la dimension matérielle de la médiation ni le rôle des usagers dans la réalité médiatique. L'approche structuraliste, que propose Éliséo Véron, en tient compte.

Un média, c'est : [...] un ensemble constitué par une technologie PLUS ${ }^{11}$ les un mediques sociales de production et d'appropriation de cette technologie, pratiques sociales de production et d'approprianion de cer accès, qui est lorsqu'il y a accès public (quelles que soient les conditions de cet acces, qui est généralement payant) aux messages ${ }^{12}$.

Comprise comme l'application pratique de la connaissance à un champ particulier et comme la capacité de faire qui en découle, la technologie concerne aussi culier et commes la les pratiques anctions qui en font, selon l'expression de Lars Elleström, des qualités et fonctions qui en font, selon lias qualifiés ${ }^{13} »$, les arts n'en sont donc pas moins des médias. Ce que Véron rassemble sous l'expression « technologie PLUS les pratiques » correspond sensiblement à l'idée générale mais hautement problématique de « dispositif » qui est capitale dans l'élaboration de la théorie intermédiale. Terme décisif dans la pensée de Foucault, le dispositif n'est pas sans lien avec celui d'institution qu'a développé et popularisé Pierre Bourdieu. Giorgio Agamben en offre une définition synthétique, articulée en trois points :

1. [Le dispositif est] un ensemble hétérogène qui inclut virtuellement chaque chose, qu'elle soit discursive ou non : discours, institutions, édifices, lois, mesures de police, propositions philosophiques. Le dispositif pris en lui-même est le réseau qui s'établit entre ces éléments.

2. [L]e dispositif a toujours une fonction stratégique concrète et s'inscrit toujours dans une relation de pouvoir.

3. [C]omme tel, il résulte du croisement des relations de pouvoir et de savoir ${ }^{14}$

Dans une perspective intermédiale, la question des socialités - donc des usagers, ce qui relève de la sociomédialité - est évidemment centrale et les intermédialistes comme les archéologues des médias accordent une importance considérable aux usages, aux protocoles et aux valeurs qui font le succès ou l'insuccès d'un média. Ces éléments font bien entendu partie intégrante du dispositif, comme les appareils, les langages (informatiques ou autres), les formats, les interfaces, les intrafaces, etc.

11.- Souligné par l'auteur.

12.- Éliséo Véron, « De l'image sémiologique aux discursivités 》, Hermès, « Espaces publics en images $\gg, n^{\text {os }} 13-14,1994, p .51$

》ia 》, Lars Elleström, « The Modalities of Media: $A$ Model for Understanding Intermedial Relations $\gg$, in Lars Elleström (dir.), Media Border Multimodality and Intermediality, New York, Palgrave Macmillan, 2010, p. 12.

14-- Giorgio Agamben, Qu'est-ce qu'un dispositif?, coll. « Rivages poche/Petite bibliothèque trad. de l'italien de Martin Rueff, Paris, Payot Rivages, 2007, p. 10-11.
L'attention grandissante portée au dispositif - et en particulier à sa base matérielle et technologique -, entendu dans ce sens très élargi, coïncide avec le passage de l'« intermédia » à l'《 intermédialité ». Selon toute vraisemblance, le mot Intermedialität - d'où découlent les termes intermédialité et intermediality serait d'abord apparu chez les philosophes des médias (dont Ole HansenLöve ${ }^{15}$, en 1983) avant de se répandre dans le champ des études médiatiques où l'Allemand Jürgen E. Müller, figure dominante de cette période fondatrice de l'intermédialité, en a popularisé l'usage. Il rappelle ainsi la conjoncture du déplacement historique du terme, du champ des arts à celui de la théorie médiatique.

À cette époque (les années 1980), les théories et les histoires des médias avaient tendance à isoler les médias et l'idée banale qu'aucun média ne pouvait être considéré comme une « monade » nous a amenés, quelques chercheurs et moi-même, à nous pencher sur les processus complexes et entrelacés qui étaient à l'œuvre dans les interactions ou lors des rencontres entre les médias. La étaient d'intermédialité était basée sur la conviction qu'il n'y a pas de média pur et que, d'une part, tout média intègre des structures, des processus, des principes, des concepts, des questionnements issus d'autres médias apparus au cours de l'histoire des médias en Occident, et, d'autre part, tout média joue avec ces éléments ${ }^{16}$

Ainsi, c'est au sein des études médiatiques allemandes, les Medienwissenschaften, et non dans les avant-gardes artistiques, que s'est produite cette avancée significative, au plan théorique, avec comme effet majeur le déplacement de l'intérêt des chercheurs des médias vers les relations entre les médias.

\section{La remédiation, concept phare de la période médiatique de l'intermédialité}

Le terme même d'intermédialité n'est pas sans causer problème. Il est historiquement marqué - on pourrait même dire qu'il date, portant la trace de la conjoncture qui l'a fait naître. Formé du préfixe « inter » et du radical « média », il a d'abord suscité la dérision : les intermédialistes s'intéressaient donc à ce qui se trouve entre ce qui est entre, c'est-à-dire pas grand-chose... Si la première

15. - Ibid, p. 15

16.-INotre tradn

and histories and « At that period [the 80s], the isolating tendencies of media theories have motivas and the rather banal fact that no medium could be considered as a "monade" cate and compled me and also some other scholars to direct our attention towards the intrimediality complex processes of media interactions or media encounters. The notion of interintegrate was based on the assumption that there are no pure media and that media would integrate structures, procedures, principles, concepts, questions of other media which have ürgen E. Mïll the history of Western media and would play with these elements », Nodus Publik, Intermedialität. Formen moderner kultureller Kommunikation, Münster, nouvelle approtionen, 1996, p. 70. Voir aussi, du même auteur : « L'intermédialité, une la vision de printemps 2000 , p. $105-134$. Cinémas. Revue d'études cinématographiques, vol. 10, $\mathrm{n}^{\mathrm{os}} 2-3$, 
proposition est vraie - entre ce qui est entre -, la seconde, qui est évidemment fuusse illustre bien la défiance quasi généralisée du milieu scientifique de l'époque. Il n'en reste pas moins que la question de l'« entre » - le in-between en anglais et la conception que s'en font les intermédialistes sont à la base même de l'éclosion de la pensée intermédiale.

Si cet intérêt pour l'entre n'a pas été compris, c'est en partie dû à la confusion que crée le préfixe «inter ». L'intermédialité a été dès le début et de façon spontanée associée - elle l'est encore - aux concepts d'intertextualité, d'interdiscursivité, d'interdisciplinarité et d'intersubjectivité, dont elle ne partage pourtant que le préfixe. On avait ainsi tendance à l'inscrire dans un télos rassurant ${ }^{17}$ mais en bonne partie erroné. Assimiler l'intermédialité à cette logique de l'inter héritée de la sémiotique, c'était en effet la dénaturer. Et cette confusion était aggravée par la juxtaposition du radical «média » qui ne faisait qu'en dédoubler le sens. Cela mérite quelques éclaircissements.

Dans une étude qu'il consacre à la notion de $\left\langle\right.$ medium $^{18} \gg-$ ce qui est au centre, au milieu -, Jacques Rancière insiste particulièrement sur la relation d'antériorité - la « relation préalable » - qu' instaure le principe de l'inter. Pour qu'il y ait un entre, affirme Rancière, il faut qu'il y ait déjà deux termes - en l'occurrence, deux textes, deux disciplines, deux médias - mis en relation dans l'espace, dans le temps, dans la logique (bref dans un cadre ou dans un système) dont cet entre devient l'entre.

Le mathématicien, philosophe et psychiatre français Daniel Sibony a poussé plus loin la réflexion dans son ouvrage Entre-deux. L'origine du partage, publié en 1991, qu'on peut considérer comme une première tentative de théorisation de cet entre dont, précisément, l'intermédialité se distingue mais auquel elle a été et est encore associée. Sibony situe le concept de l'entre, qu'il nomme justement l'« entre-deux », dans le prolongement de la théorie poststructuraliste de la différence dont il serait le dépassement : «Nous avons vécu et pensé jusqu'ici sous le signe de la différence », mais « les faits, les événements - très mouvementés ces temps-ci... obligent à ne plus se contenter du repère de la différence ${ }^{19} \gg$. Pour

17.- Voir Éric Méchoulan, « Intermédialités : Le temps des illusions perdues 》, Intermédialités. Histoire thérie des arts, des lettres et des techniques, « Naître 》, $\mathbf{n}^{\circ} 1$, printemps 2003 p. 11.

18.- Voir Jacques Rancière, « Ce que "medium" peut vouloir dire : l'exemple de la photographie », Revue Appareil, $\mathrm{n}^{\circ} 1,17$ février 2008, < http://appareil.revues.org/135> (consultation le 30 juin 2014). Dans cet article comme dans l'ensemble du présent ouvrage, nous avons opté, à chaque fois que cela était possible, pour la paire média et médias plutôt que pour la forme latine medium et media pour la simplêtaison que le terme medium revêt ê français d'autres significations que celle à laquelle nous le rattachons.

19.- Daniel Sibony, Entre-deux, L'origine en partage, coll. « La couleur des idées », Paris Éditions du Seuil, 1991, p. 9. Sibony souligne.
Sibony, si l'idée de la différence est foncièrement juste, elle s'avère limitée et sa pertinence reste infime, d'où son élargissement à l'entre-deux comme « opérateur [...] efficace ${ }^{20} \gg$ qui permet d'aborder la complexité des phénomènes à l'œuvre dans l'« entre », phénomènes que Sibony qualifie d'《 épreuves », tant l'entredeux est un espace de tensions et de déchirements.

Peut-être les épreuves d'entre-deux se ramènent-elles à des mouvements plus ou moins riches où une identité tente de recoller ses morceaux, de s'intégrer elle-même (en croyant s'intégrer à d'autres), de s'assumer comme une tenue d'Arlequin dans le cirque du monde. [...] Mais au-delà des recollements que l'entre-deux actualise, là où il prend toute sa force c'est lorsque dans son immense foisonnement, il apparaît comme une figure de l'origine, [...] qui suppose ou implique autre chose qu'un ressassement dans l'« entre-deux $\ldots$ imi suppose

Et c'est précisément ce principe d'origine, dont on perçoit la dimension nostalgique - et la vision du monde qu'elle traduit -, qui cause problème en intermédialité. C'est même la cause de la première rupture épistémique majeure que produit l'intermédialité et qui la distingue radicalement des autres figures de linter évoquées précédemment. Cette rupture porte à la fois sur la nature de des deux $\gg$ qui belisent $\alpha$ lentre-deux $\gg$ et sur deux » qui balisent « l'entre-deux » et sur le rapport d'antériorité qui y es attaché. Si le « medium », comme le définit Rancière, est un entre, l'intermédialité s'intéresse à un entre qui se trouve entre d'autres entres. En ce sens, il faut prendre au pied de la lettre les boutades de ceux qui se moquaient : oui, l'intermédialité s' intéresse bien à ce qui se trouve entre ce qui est entre. C' 'est beaucoup c'est immense, puisqu'il n'y a que cela ; et c'est complexe parce que ces entres sont dynamiques, animés, en changement constant! La disparition de points fixes, de pôles préalables, dans cette nouvelle configuration n'a pas qu'un effet déstabi-
lisant, elle exclut tout principe d'antériorité et fait éclater le mythe de l'origine.

Les premiers intermédialistes avaient conscience de la nouveauté de leur approche, mais ils n'en ont pris toute la mesure que très progressivement, comme en témoigne l'examen rétrospectif de l'évolution de la pensée intermédiale que proposait Jens Schröter en 2012.

[N]ous dev[i]ons reconnaitre que ce ne sont pas les médias individuels qui son premiers et qui se déplacent ensuite intermédialement de l'un vers l'autre, mais que c'est l'intermédialité qui est première et les «monomédias $\gg$ clairement séparés sont le résulat de blocages, incisions et tionnels et réfléchis ${ }^{22}$

22. - Notre trad 16. Sibony souligne.

and then move toward we have to recognize that it is not individual media that are prima and that the clearly separated "monomedia" are the result of purposeful and institutionally 
On aura noté que Schröter ne va pas ici jusqu'à remettre en question la figure de l'origine défendue par Sibony ni l'existence des médias, il se contente d'en inverser l'ordre, ce qui est déjà considérable : les médias sont le produit et non la source des relations intermédiales.

C'est cette logique qui domine la période médiatique de la réflexion intermédiale et que Jay David Bolter et Richard Grusin résument par la formule choc « un média est ce qui remédie ${ }^{23} \gg$ dans leur célèbre essai. Remediation. Understanding New Media qui, rappelons-le, paraît en 2000 , soit une quinzaine d'années après l'émergence de la réflexion intermédiale, est un moment clé de la jeune histoire de l'intermédialité dont il propose une première modélisation. C'est aussi un ouvrage charnière qui dresse un bilan des avancées de l'intermédialité à l'aube $\mathrm{du} \mathrm{XXI}^{\mathrm{e}}$ siècle tout en ouvrant sur des perspectives qui se trouvent au cœur de la pensée intermédiale actuelle. S'appuyant sur l'idée de Marshall McLuhan, relayée par Kittler et bien d'autres, selon laquelle « le contenu d'un médium, quel qu'il soit, est toujours un autre médium ${ }^{24} \gg$, les deux auteurs soutiennent que :

[r]out média s'approprie les techniques, les formes et la signification sociale d'autres médias et tente de rivaliser avec eux ou de les refaçonner au nom du réel. Dans notre culture, un média ne peut jamais opérer isolément parce qu'il doit entrer en relation, de respect ou de rivalité, avec d'autres médias ${ }^{25}$

Le principe de remédiation va au-delà des intuitions des premiers intermédialistes en ce sens que, tout en restant centrés sur la genèse des médias, Bolter et Grusin en excluent les questions d'antériorité et d'origine. En effet, si un média est bien ce qui remédie, cela signifie qu'il n'y a pas de première remédiation. Lisa Gitelman illustre cette logique déconcertante, qui va à l'encontre de la tradition et de l'école de pensée défendue par Sibony, par une expression dont on reconnaît l'écho derridien : un média est « toujours déjà nouveau ${ }^{26} »$. Le modèle développé par Bolter et Grusin, sans renier le média, l'inscrit dans un mouvement perpétuel - la remédiation - et met clairement l'accent sur ce que le média produit et sur ce qui le produit. En ce sens, ils confirment l'idée de Chiel Kattenbelt selon laquelle

caused blockades, incisions, and mechanisms of exclusion », Jens Schröter, « Four Models of Intermediality », in Bernd Herzogenrath (dir.), Travels in Intermedia [lity]: ReBlurring the Boundaries, Hanover (New Hampshire), Dartmouth College Press, 2012, p. 30

23. - Notre traduction] « a medium is that which remediates », Jay David Bolter et Richard Grusin, op. cit., p. 65.

24. - Marshall McLuhan, Pour comprendre les médias. Les prolongements technologiques de l'homme, trad. de l'anglais de Jean Paré, Montréal, 1969 [1968], Éditions HMH, p. 24

25.- [Notre traduction] « [A medium] appropriates thechniques, forms and social signif cance of other media and attempts to rival or refashion them in the name of the real. Ame dium in our culture can never operate in isolation, because it must enter into relationships for 65

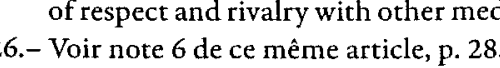

l'intermédialité serait actuellement la forme la plus radicale de la performativité ${ }^{27}$. Ceci dit, Bolter et Grusin restent clairement pris dans le paradigme du représentationnel. La remédiation se ferait « au nom du réel »! On s'étonne de cette fixation à une époque où ce paradigme devient de moins en moins défendable particulièrement dans le champ théâtral. Cette question est importante puisque le principe du rapport au réel, que défendent les deux auteurs, a des ramification profondes dans divers aspects de la dynamique intermédiale. Essentiellement, il accrédite l'idée que le média est une entité discrétisédiale. Essentiellement, i bonne partie fondée sur un dispositif - qui a une base matérielle -, se limite à C en passeur, transformateur ou producteur de données.

C'est clairement en ce sens qu'il faut comprendre le concept d'immédiateté - immediacy - que les deux auteurs lient à certaines formes de médiation. Il y a immédiateté, soutiennent-ils, quand la médiation est imperceptible à l'usager qui pourrait donc se croire en présence de ce qui, dans les faits, lui est seulement représenté. L'immédiateté est donc liée à la transparence - transparency - du média : le média s'efface, son dispositifest occulté Nous sommes ici en plein illugédia : le dans une logique qui ressortit à la mimesis et qui reprend les arguments traditionnels sur lesquels l'industrie médiatique a fondé ses stratégies de marketing depuis plus d'un siècle, telles les promesses de « fidélité » et de « haute fidélité » - on ne sait pas vraiment à quoi - claironnées par les compagnies de disque dès le début du XX $X^{\mathrm{e}}$ siècle $^{28}$. C'est le début du XX ${ }^{\mathrm{e}}$ siècle ${ }^{28}$. C'est en ce sens qu' il faut comprendre l'expression « au nom du réel » mentionnée plus tôt par Bolter et Grusin.

Si ces deux penseurs restent très influencés - et limités - par le modèle mimétique, ils réalisent bien qu'il existe d'autres formes de médiations qui ne renvoient pas à un réel tangible, autonome, qui se trouverait à l'extérieur, détaché et indépendant du processus de médiarion. qui se trouverait à lextérieur, détaché diateté, ils développent donce de médiation. Parallèlement à la logique de l'immépremière est expliquée de foch hypermédiateté - hypermediacy. Mais autant la est laboric expliquée de façon limpide, autant l'argumentaire appuyant la seconde deux auteurs è lacunaire, ce qui illustre autant sa complexité que la difficulté des Phyperméd larticuler. Plus diversifiée dans ses manifestations que l'immédiateté, aspect du concepté ne convoque donc pas de réel externe. Pour illustrer ce premier he renvoie - en on pourrait suggérer une analogie avec la peinture abstraite qui (par opposition principe - à rien d'autre qu'à elle-même et à ce qui la constitue d'artifice - don à la peinture figurative qui, en dépit de son dispositif créateur médiateté est plus querspective -, donne une impression de réel). Mais I'hyperMédiateré est plus que cela. L'image hypermédiale à laquelle recourent Bolter et

$27 .-$ Voir son article, in

28. Le slon article, infra, p. 101

Saths doutele plus percutant. 
Grusin dans leur démonstration est celle d'une page du World Wide Web (non Grusin dans le son dans leur exemple). Cette page se compose d'éléments dispaaccompagnée de son dans leur exes icônes, des photos, des images de toutes sortes, rates - des lettres, des ch co co crabitent sur la surface de l'en un système fixes et en mouvements $-q u i$ de relations complexes et en perpétuelle transformation. Contrairement à l'effet de transparence qui, rappelons-le, tend à supprimer toute perception de la médiation, la page web offre le spectacle d'une médiation à l'œuvre. Au principe de transparence lié à l'immédiateté s'oppose ici celui d'opacité - opacity. Alors que la transparence 作 rén de médiation, au plaisir qu'ils prendraient à les voir en mouvement.

La logique de l'hypermédiateré amène l'artiste (ou le programmeur multimédia ou le web designer) à rendre l'usager conscient de la présence du média et à faire en sorte qu'il tire plaisir de cette prise de conscience ${ }^{29}$.

À ce deuxième aspect de l'hypermédiateté s'en ajoute un troisième. La page web, qui est une fenêtre - a window -, n'est pas un seuil métaphorique, comme l'écran du cinéma traditionnel ou de la télévision qui, une fois franchi, permet d'accéder à une réalité au-delà de l'écran : la fenêtre que constitue la page web ne renvoie qu’à d'autres fenêtres, jamais à une réalité en trois dimensions qui aurait ou pourrait avoir une existence hors d'elle. Richard Lanham synthétise cette différence par un changement de préposition : looking at plutôt que looking through ${ }^{30}$ (regarder et regarder à travers). Bolter et Grusin résument la chose ainsi :

L'hypermédiateté contemporaine offre un espace hétérogène dans lequel la représentation n'est pas conçue comme une fenêtre sur le monde mais plutôt comme étant une fenêtre en tant que telle - avec des fenêtres qui s'ouvrent sur d'autres représentations ou d'autres médias. La logique de l'hypermédiateté multiplie les signes de médiation et, en ce sens, elle tente de reproduire le sensorium de l'expérience humaine ${ }^{31}$.

Cette dernière remarque préfigure les grands débats qui animent le champ de a théorie intermédiale à partir de 2005, avec, comme principal enjeu, l'abandon

29.- [Notre traduction] « the artist (or multimedia programmer or web designer) strives make the viewer acknowledge the medium as a medium and to delight in that acknowle gement $\gg$ Jay David Bolter et Richard Grusin, op. cit., p. 41-42.

30. Cité par Boler et Grusin, op.cit. p. 41. Voir aussi Richard Lanham, The Electronic Word Demé par Bolter et Gress, 1993, 285 p. Democracy, Technology, and the Arts, Chicago, Univcrsity of Chicago Press, 1993, $285 \mathrm{p}$.

31.- [Notre traduction] « [C]ontemporary hypermedia\& $\mathbf{z}$ offers a heteregoneous space, which representation is conceived of not as a window on to the world, but rather as « wir dowed $\gg$ itself - with windows that open on to other representations or other media. The logic of hypermediacy multiplies the signs of mediation and in this way tries to reprodue the rich sensorium of human experience », Jay David Bolter et Richard Grusin, op. ct p. 34 . graduel du concept de média au profit de celui de médiation. Du point de vue du théâtre, cela correspondrait au passage du représentationnel au présentationnel, concepts qu'ont développés Tracy C. Davies et Thomas Postlewait à propos de la théâtralité $e^{32}$ - le représentationnel participant de la logique mimétique alors que le présentationnel correspondrait à celle de la performance où, en principe, on ne représente pas la réalité, on y est.

L'essai de Bolter et Grusin marque l'apogée de cette première période de la réflexion intermédiale, il en révèle aussi les limites. Pour Alexander R. Galloway, auteur de The Interface Effect, « I'argument de la remédiation [...] est si rempli de trous qu'il vaut probablement mieux tout jeter ${ }^{33} \gg$. Le jugement est sans doute sévère mais il n'est pas sans fondements. C'est à l'examen de ces limites er à d'autres modèles de dynamique intermédiale que se sont consacrés les chercheurs depuis une dizaine d'années, alors que s'ouvrait la période postmédiatique de l'intermédialité. Commençons par les limites.

\section{La période postmédiatique de l'intermédialité}

\section{Les limites du modèle de remédiation}

L'une des limites du modèle remédiant découle de l'idéologie de ses concepteurs depuis McLuhan. Les premiers grands chantiers intermédiaux se sont centrés sur des « remédiations réussies » qui ont abouti à la formation de « nouveaux médias à succès (tous électriques ou numériques) avec, pour exemples emblématiques, le cinéma, la radio, la télévision et la vidéo numérique. Ce n'est pas un hasard. Cet intérêt s'explique par une conception darwiniste de l'évolution des médias fondée sur la notion de progrès : on peut en effer présumer que, de réussite en réussite et « au nom du réel », nous approchons sans cesse du média absolu et universel, celui qui sera capable de tout médiatiser. C'est le mythe de la « boîte noire $\gg$ - The Black Box - sur lequel nous allons revenir

Cette vision optimiste et linéaire de l'évolution des médias est cependant contredite par la réalité. Si nous allons vers une fidélité - ou une transparence lens cesse plus accrue, comme le répète d'ailleurs l'industrie, comment expliquer partir de téléphones portablese fidélité tels les webdocumentaires réalisées à clair que air que, contrairement à ce que pourrait laisser croise le modèle remédiant,

32. Voir Tracy C. Davis

Theory $\gg$, Cambrid et Thomas Postlewait (dir.), Theatricality, coll. « Theatre Performance 13. [Notre traduction] Cambrige, Cambridge University Press, 2003, $243 \mathrm{p}$.

best to toss it whe remediation argument $[. .$.$] is so full of holes that it is probably$ 1.- Un enregistrement MP3, Alexander R. Galloway, op. cit., p. 20.

cotrespondant. Voir à3 n'occupe que $15 \%$ de l'espace de l'enregistrement analogique Eormat, Durham/Londres, Vos l'essai édifiant de Jonathan Sterne, MP3. The Meaning of a 
tout processus de remédiation n'entraîne pas l'apparition d'un nouveau média. Il crée aussi des perdants, produit des ratés, des déchets, déclenche des réactions imprévisibles et chaotiques. Que reste-t-il des tentatives avortées de remédiation, qu'advient-il des médias qui, contrairement au théâtre, par exemple, ne survivent pas à leur(s) remédiation(s) - par le cinéma, par la radio et la télévision - ou y survivent mal, perdant en influence, cessant d'être mainstream ? Cet aspect y surentel de la remédiation, qui porte sur ce que Charles Acland nomme les médias résiduels ${ }^{35} \gg$, est tout à fait ignoré par Bolter et Grusin. Tout indique pourtant que les cas de remédiations non abouties - entendons par là qu'ils n'aboutissent pas à des médias - sont la règle et non l'exception.

C'est ans doute la prise de conscience de toutes ces autres actions remédiantes qui explique que, de l'analyse des grandes réussites qui ont marqué la recherche intermédiale jusqu'au milieu des années 2000 , nous sommes passés à celle de ces résistances et de ces restes qui ne sont pas moins fertiles et instructifs. Les études ur le son au théâtre font partie de ces nouveaux chantiers qui nous permettent de i de retours en arrière, d'accidents - dont la généalogie des médias, forcément linéaire, qui inspire les premiers intermédialistes, pouvait difficilement rendre compte.

Sibony liait l'entre-deux à l'origine, mais il le présentait aussi comme un lieu d'épreuves et, effectivement, les processus de remédiation entraînent parfois d'épreuves et, effectivement, les processus de remédion violentes qui relèvent du - mais pas toujours - des réactions plus ou moins vion peut comprendre comme principe général de « résistance médiatique » qu'on peut comprent, lui aussi, du une tentative de contre-remédiation et qui est totalement absent, lui aussi, du champ d'analyse de Bolter et Grusin. Cette résistance est un mécanisme de défense qui se déclenche lorsque des fondements de la médiation sont ou, plus exactement, semblent mis en danger par l'intrusion d'un nouvel élément. Le mécanisme de résistance a pour effet d'empêcher, d'éviter, de retarder l'introduction de ce nouvel élément ou de le rejeter après qu'il se soit introduit. Dans ce dernier cas, on pourrait parler de « dé-remédiation » ou, plus simplement, de « démédiation ». Les reculs observés dans l'exploitation des sons enregistrés au théâtre en sont un exemple. L'analyse de devis techniques et de relevés de mise en

35. Notre traduction, «[r]esidual [m]edia », voir Charles R. Acland (dir.), Residual Media Londres/Minneapolis, Universiry of Minnesota Press, 2007, $401 \mathrm{p}$.

L' les rapports du théâtre et des technologies depuis Meyerhold. Voir son ouvrage Entangled. Technology and theatre et des technologies depuis 2010,460 pages.

37.- Voir à ce sujet Jean-Marc Larrue, « Sound Reproduction Technique in Theatre: A Case o

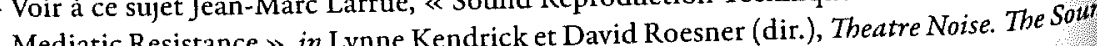
of Performance, Cambridge, Cambridge Scholars, 2011, p. 14-22. scène ${ }^{38}$ montre que le « son technologiquement reproduit », qui avait fait une entrée remarquée sur les scènes théâtrales, en a été ensuite progressivement exclu, et rien ne permet de penser que ce rejet ne tenait qu'au déclin de son pouvoir attractif (à titre de curiositié ou de nouveauté). Bien entendu, il n'y a pas d'essentialisme médiatique. Lorsqu'un média résiste à l'intrusion d'une nouvelle technologie - en général, c'est de cela qu'il s'agit -, il ne se comporte pas comme un organisme vivant mobilisant des anticorps pour chasser ou détruire l'intrus. La résistance est toujours le fait du milieu - constitué d'hommes et de femmes - où opère le média, elle relève de représentations, elle est liée à un discours. Pour rester dans le domaine du son reproduit, on constate que le microphone, longtemps rejeté par le théâtre - où son usage est cependant de plus en plus fréquent - a été d'emblée intégré par la scène du cabaret (la chanson). La résistance médiatique obéit à une double logique : d'une part, c'est une réaction à la propension de toute pratique médiatique, artistique ou non, à intégrer à son action médiatrice des savoirs, des techniques, des technologies, des valeurs, des comportements, des protocoles qui lui sont étrangers et, d'autre part, c'est un frein à la propension de ces divers éléments à envahir cette pratique. La résistance médiatique met aussi en lumière le fait que la remédiation ne se joue pas qu'entre médias, elle concerne aussi des éléments - encore une fois, souvent des technologies - sans attache médiatique comme on a pu l'observer lors de la révolution numérique. Elle n'est pas non plus unidirectionnelle. On doit retenir de cela qu'il n'y a pas que les médias qui remédient et sont remédiés, et que la remédiation entraîne toutes sortes d'effets, dont son contraire, la résistance médiatique.

Parmi tous ces effets, l'un est assez inattendu, il a trait à la question de l'opacité. Selon Bolter et Grusin, il y a opacité lorsque l'usager perçoit la médiation. Prenons lexemple du micro au théâtre. Pour des raisons que nous détaillons plus loin - la question centrale de la « présence »-, le théâtre, comme l'opéra, a d'abord exclu le recours au micro sur scène alors que trois pratiques médiatiques, le disque, la radio, le cinéma parlant (auxquels on pourrait ajouter le cabaret), se sont dévéloppées et ont connu le succès qu'on sait grâce à lui. La radio a joué un rôle moteur dans cette évolution. Son micro a véritablement révolutionné l'art de la parole publique, provoquant une formidable explosion des styles vocaux. Avec la radio, insiste Jacob $S_{\text {mith }}^{39}$, le public a découvert que les paroles intimes, les conversations de tous les jours pouvaientêtre hissées au rang de paroles publiques et reproduites telles quelles (du moins c'est ainsi qu'on le percevait) dans leur infinie diversité, sans exagération,

38. Voir it ce sujet Jean

Typermédialités du théátre Larrue et Giusy Pisano (dir.), Les archives de la mise en scène. Jacob Smith, Vocal Tractse, Lille, Presses Universitaires du Septentrion, 2014, 422 p. Tress, 2008, vocal Tracks. Performance and Sound Media, Berkeley, University of California 
sans les effets qu'on leur connaissait sur scène ${ }^{40}$, en toute transparence. C'était le eners, célèbres pour leurs voix murmurées. Prenant acte de ce hangement et saisissant toute la mesure de la puissance persuasive de cette radio in intimiste, Franklin Delano Roosevelt instaurait, des 1929, alo économique, la pratique des Fireside Chats - les « Causeries au coin du feu » qu'il maintint jusqu'en 1944 et dans lesquels il entretenait en toute simplicité ses concitoyens des grands problèmes de l'heure. La radio sonnait vrai. Et le public ne s'y trompait pas. La comparaison avec le théâtre, autre lieu de parole publique, était inévitable et c'est ainsi, insiste $S$ mith, que les « conventions vocales de la scène $\operatorname{dev}\left[\right.$ inrent] bien trop perceptibles à beaucoup d'auditeurs ${ }^{41} \gg$

Certe nouvelle auralité, créée par la radio, faisait ressortir l'artificialité de de théâtre, fondées depuis toujours sur la la voix et de la diction des acteurs de theâtre, fondéc projection ou le volume et l'éloquence. Jusqu a la fin du XIX belle voix était une l'apparition des technologies de reproduction du son, une belle voin étaique - le voix forte. La comparaison mettait en évidence de « vérité », défendait une parole « dénaturée »! théâtre - qui, sous prétexte de « vérité », défendait une parole « dénaturée »!

Resitué dans une logique remédiante, ce phénomène consiste à rendre opaque un aspect de la médiation qui avait été préalablement naturalisée, contribuant rendre celle-ci partiellement ou totalement transparente. Il s'agit d'une manifestation claire du phénomène d'opacification. Dans le cas évoqué plus tôt, un média, en l'occurrence la radio, a rendu opaque la médiation d'un autre, le théâtre. Ainsi, contrairement à ce que laissent entendre Bolter et Grusin, l'opacité ne relève pas de la stratégie d'un média et de sa dynamique évolutive mais d'une conjoncture sur laquelle il a, en fin de compte, assez peu de prise.

Les mécanismes de résistance médiatique et d'opacification enrichissent le modèle de remédiation tout en soulignant ses limites. Les concepts d'hypermédialité, de convergence et de transmédialité, qui sont au cour de la réflexion inter médiale depuis dix ans, ont des conséquences plus profondes.

\section{Hypermédialité et transmédialité :}

\section{d'autres modéles de dynamiques intermédiales}

Le concept d'hypermédialité a émergé du champ des études théâtrales au milie des années 2000. D'un point de vue intermédial, qu'on pourrait paradoxalemen qualifier de traditionnel, le théâtre est un média. Mieux encore, c'est la pratique intermédiale par excellence dans la mesure où il est lui-même fait de médias interagissant les uns avec les autres - la musique, la scénographie, les pratiques plastiques, la danse et la gestuelle, la dramaturgié, etc. -, comme interagissent les

40.- Ibid., p. 100.

位 dible as conventions to many listeners $\gg$, ibid, p. 94 différents éléments qui composent la page web. Selon la terminologie utilisée par Bernd Herzogenrath, le théâtre serait un « intermedium ${ }^{42} \gg$, selon celle de Jen Schröter, ce serait un «super medium ${ }^{43} \gg$, mais Chiel Kattenbelt va plus loin encore : le théâtre est un hypermédia ${ }^{44}$.

Le film, la télévision, la vidéo numérique sont des médias à base technologique qui peuvent enregistrer et reproduire tout ce qui est visible et audible, en fonction de leurs capacités techniques, mais ils ne peuvent pas incorporer d'autres médias sans les transformer et les soumettre à leur propre médialité. Un média peut aller jusqu'à remédier (Bolter et Grusin) d'autres médias, ce qui implique, à terme, un refaçonnement de ces derniers. Il est clair que le théâtre n'est pas un á terme, la même façon que le film, la télévision et la vidéo numérique sont des médias. Toutefois, bien que le théâtre ne puisse pas enregistrer [le visible et l'audible] comme les autres médias, il peut, de la même façon qu'il incorpore les autres arts, incorporer tous les médias à son espace performatif. C'est en raison de cette capacité singulière que je considère le théâtre comme un hypermédia ${ }^{45}$.

Il faut donc prendre le terme hypermédia en son sens littéral de média fédérateur et non de média assimilateur ou transformateur. C'est un « archimédia » ou «média de médias », « home to all », foyer, espace de rencontre interartiale et technologique « où les formes artistiques du théâtre, de l'opéra et de la danse se rencontrent, interagissent et intègrent les médias cinéma, vidéo et les nouvelles technologies ${ }^{46} \gg$. Cette hospitalité se reflète dans le traitement singulier que le théâtre réserve aux autres objets médiatiques, aux technologies,

42.- Bernt Herzogenrath, « Travels in Intermedia[lity]. An Introduction », in Bernd erzogenrath (dir), Travels in Intermedia[lity]. ReBlurring the Boundaries, Hanover (New Hampshire), Dartmouth College Press, 2012, p. 2.

43.- Jens Schröter, op. cit., p. 20. Nous soulignons.

44.- Chiel Kattenbelt, « Theatre as the Art of the Performer and the Stage of Intermediality 》, in Freda Chapple et Chiel Kattenbelt (dir.), Intermediality in Theatre and 'Performance, coll. « Themes in Theatre », Amsterdam/New York, Rodopi, p. 29.

- Notre traduction $] \ll[F] i l m$, television and digital video are technology-based media that an record and play back everything that is visible and audible, within their specific that fensitivity, but they cannot incorporate other media withour transforming them er the conditions of the specificity of their own mediality. At the very most emediate (Bolter and Grusin 1999) other media, which Clearly, theatre is and Grusin 1999) other media, which implies in the end a refashioning. However, although a medium in the way that film, television and digital video are media. incorporate the otheatre cannot record in the same way as the other media, just as it can is in this cather arts, so it can incorporate all media into its performance space. It as the A capacity that I regard theatre as a hypermedium », Chiel Kattenbelt « Karte [Notre trelt (dir.), op. cit., p. 37.

in integrate with the «edia of the art forms of theatre, opera and dance meet, interact and Chapple et Chiel Kedia of cinema, television, video and the new technologies », Freda Chapple et Chiel Kattenbelt, « Key Issues in Intermediality and Performance », in Freda 
aux autres arts et médias qui entrent dans le processus de la création ou dans celui de la représentation théâtrale.

Le concept d'hypermédialité découle évidemment de celui d'hypermédiateté mais il le déborde. Il est d'autant plus séduisant qu'il exclut la question de la représentation - et du rapport au réel - dans la mesure où, pour reprendre la métaphore de la «fenêtre » évoquée par Lanham, il ne nécessite pas qu'on tranche entre « regarder »-looking at - et « regarder à travers »-looking through. Mieux encore, il permet les deux actions simultanément, ce qui convient bien, on l'admettra, aux pratiques théâtrales actuelles et à l'approche performative, fondée sur l'expérientiel, qu'on en a. Le principal impact du concept d'hypermédialité proposé par Chiel Kattenbelt sur la pensée intermédiale a été de révéler l'existence d'autres régimes d'interactions médiatiques et technologiques qui ne relèvent pas de la logique remédiante et qui n'en ont pas la violence cannibale.

L'année même - 2006 - où Chiel Kattenbelt développe le concept d'hypermédialité, Henry Jenkins s'attaque à une idée maîtresse de la généalogie des médias, sous-jacente au concept de remédiation : la convergence médiatique. Cette idée n'est pas nouvelle, on en observe une première expression célèbre dans le projet wagnérien de Gesamtkunstwerk - l'œuvre d'art totale -, mais elle s'est véritablement et durablement imposée comme une évidence à mesure que se déployaient les médias électriques dans le premier tiers du $\mathrm{XX}^{\mathrm{e}}$ siècle. L'avènement du cinéma parlant en est une des plus célèbres et des plus mémorables illustrations. Le parlant résulte en effet de la convergence longtemps annoncée des technologies de reproduction du son et de l'image qui, jusque-là, s'étaient développées séparément. Trois facteurs principaux seraient à la base de la convergence médiatique : les progrès continus des diverses technologies, le déterminisme technologique qui les amènerait à fusionner, la recherche de la plus grande transparence possible. Le principe de convergence, qui serait actif dans toute dynamique médiatique, le serait « au nom du réel » comme le rappellent les slogans successifs de l'industrie que nous avons déjà évoqués : la « fidélité », la « haute fidélité », la « stéréophonie », la « haute définition », le $\ll 3 \mathrm{D} »$, etc. Ces slogans successifs seraient autant de balises sur cette route triomphale qui mènerait ultimement au média absolu et à la parfaite transparence de la médiation - au sens de Bolter et Grusin. «Tôt ou tard, selon cette logique, le contenu de tous les médias va converger dans une simple petite boîte noire [installée] dans nos salons (ou, dans le scénario de la mobilité, dans une boîte noire que nous aurons sur nous partout où nous allons $)^{47} \gg$, prévient Jenkins avec un soupçon d'ironie. La convergence médiatique serait l'ultime forme de remédiation, la

47.- [Notre traduction] « Sooner or later, the argument goes, all media content is going to flor through a single black box into our living rooms (or, in the mobile scenario, through blach
À la différence de l'hypermédia de Kattenbelt, qui est fédérateur et préserve la médialité de chaque média participant, la « boîte noire » est intégratrice, elle mêle et fusionne tout ce qui entre en elle. L'idée de convergence est si répandue et si souvent invoquée par l'industrie, qu'elle semble naturelle et inéluctable, mais ce n'est pas le cas, insiste Jenkins. Dans la réalité, on ne peut pas isoler les progrès technologiques de la conjoncture qui les roite, on ne peut pas isoler les progrè . griqe est un leurre, un argument de marketing : « la convergence renvoie à des processus, non à un but ${ }^{48} \gg$.

Une des raisons [affirme Jenkins] qui font du concept de la boîte noire une tromperie est qu'il réduit les changements médiatiques à des changements technologiques et laisse de côté la dimension culturelle ${ }^{49}$.

Or, la « convergence ne naît pas dans des accessoires médiatiques, si sophistiqués soient-ils. La convergence se trouve dans les cerveaux de consommateurs individuels et dans les interactions sociales qu' ils ont les uns avec les autres ${ }^{50}$ ». L'un des grands mérites de Jenkins est d'avoir ainsi recentré la réflexion intermé diale sur le rôle moteur des usagers (il utilise le terme « consommateurs ») e plus globalement sur le milieu sans cesse changeant d'où émergent les médias et où ils agissent. Il en vient ainsi à retourner le concept traditionnel de convergence médiatique - spontanément entendu comme convergence « technologique » en un concept de convergence globale :

où les vieux et les nouveaux médias se heurtent, où les médias de base er les grand médias s'entrechoquent, où le pouvoir du producreur de médias et le pouvoir $\mathrm{d}$ consommateur de médias interagissent de façon imprévisible ${ }^{51}$.

Comment, en effet, définir un quelconque télos dans de telles conditions ? La réponse est simple : la boîte noire est un mythe créé de toutes pièces par l'industrie médiatique pour justifier l'obsolescence rapide et le remplacement de ses produits. Mais la culture participative actuelle, marquée par le fonctionnement en réseaux et l'intelligence collective, a d'autres exigences : les usagers ne veulent pas et ne rêvent plus, si tant est qu'ils y aient déjà rêvé, d'une « relation tout-aller et

Woxes we carry around with us everywhere we go) $\gg$, Henry Jenkins, Convergence Culture 8. [Notre tdand New Media Collide, New York, New York University Press, 2008 r 2006$]$ culture 49. [Notre traduction] « convergence refers to a process, not an endpoint $\gg$, ibid 2006], p. media change to technological mat makes the black box concept a fallacy is that it reduces - [Notre traduction] « Convergenge and strips aside the cultural levels », ibid., p. 15. phisticated they may become. Convers not occur through media appliances, however soThers and through their social interacrions occurs within the brains of individual consu[Notre traduction] « where linteractions with others », ibid., p. 3. dia intersect, where the old ad new media collide, where grassroots and corporate em sumer interact in unpredictable the media producer and the power of the media concon- 
uniforme avec les contenus médiatiques. [Ils] veulent les médias qu'ils veulent où ils le veulent, quand ils le veulent, et dans le format qu'ils veulent ${ }^{52} \gg$. La convergence médiatique menait en principe vers un super média unique, la boîte noire; cette nouvelle culture participative, qui crée, selon Jenkins, une « culture de la convergence », mène précisément au contraire, la divergence médiatique. Pour s'adapter aux nouveaux comportements des usagers, l'industrie doit en effet multiplier les plateformes, spécialisant celles-ci en fonction d'usages précis, et trouver les moyens de faire circuler ses contenus médiatiques à travers elles. Cette dynamique nouvelle marque une étape décisive dans la réflexion intermédiale, la dynamique nouvelle marque une étape décisivé transmédialité qui rèle une autre forme de relations intermédiales, tout à fait différente de la remédiation et de l'hypermédialité.

Dans le troisième chapitre de son essai, consacré au phénomène de Matrix, Jenkins montre comment l'industrie, tout en continuant de célébrer l'avènement toujours plus proche du média absolu, la « boîte noire », s'est soumise à la logique de la divergence en créant des contenus médiatiques « transmédiaux », c'est-àdire adaptables aux différentes technologies médiatiques présentes sur le marché. Dans le cas de Matrix, il s'agit de trois longs métrages, d'une série télévisée de 90 minutes, de films d'animation, de multiples bandes dessinées, de jeux vidéo, etc.

Les modalités de la transmédialité sont infinies, elles vont de la simple distribution d'un contenu réputé uniforme sur différentes plateformes à la création d'une intelligence collective fragmentée dont une partie serait assumée par chacune des technologies mobilisées et dont l'ensemble, à l'image de l'arcologie de Paolo Soleri ${ }^{53}$, demeure insaisissable quel que soit le point de vue d'où on l'observe. Les enjeux, on le comprend, sont majeurs. Poursuivant la réflexion de Jenkins, Mark J. P. Wolf analyse les effets de la transmédialité sur les distinctions traditionnelles entre le monde réel et le monde médiatisé.

La transmédialité implique une certaine indépendance de son objer : plus le nombre de fenêtres médiatiques par lesquelles nous faisons l'expérience du monde est élevé, moins l'existence de ce monde est liée aux particularités d'un média précis. Ainsi, la transmédialité suppose aussi la possibilité d'un monde qui se perpétuerait dans une multitude de circonstances et de registres; et plus un monde transmédial s'entend et se voir, plus l'illusion de son poids ontologique est grande et plus l'expérience du monde se rapproche de l'expérience médiatisée du Monde Primaire ${ }^{5}$

22- [Notre traduction] $<$ one-size-fits-all relationship to media content. Consumers want the media they want where want ir, and in the format they want media the Process $\gg$, Confessio Henry Jenkins, « Convergence and Divergence: Two parts of he sar. of an Aca-Fan. The Official Wenblog of Henry Jenkins, 29 juin 2006, < http://henryjenkin org/2006/06/convergence_and_divergence_two.html > (consultation le 12 mars 201

53.- Paolo Soleri, Arcologie, la ville à l'image de l'homme, Marseille, Paranthèse, 1980.

54. - [Notre traduction] « Transmediality implies a kind of independence of its object:
Si la transmédialité ne brouille pas tout à fait la ligne de partage entre ce que Wolf appelle le Monde Primaire et le monde médiatisé, elle en rend la distinction superflue et on comprend que, dans une logique transmédiale où ce qui prime est l'expérience de l'usager - l'experiencer -, la question de la représentation perd de sa pertinence tout comme celle du jeu entre immédiateté et hypermédiateté L'accent, comme pour la convergence, est mis sur l'usager et sur son expérience
qui, elle, est toujours réelle.

\section{L'éclatement du média : avènement du postmédiatique}

La transmédialité a un autre impact majeur sur la réflexion intermédiale en remettant en cause l'existence même du média tel que le concevaient les premiers intermédialistes. Dans la définition qu'il donnait du média, Véron ne se contentait pas d'insister sur sa dimension technologique, il faisait de la technologie l'élément fondateur du média : « un ensemble constitué par une technologie PLUS [d] es pratiques sociales de production et d'appropriation ${ }^{55} »$. Le modèle de la $\ll$ double naissance », qu’André Gaudreault et Philippe Marion ont dégagé de leurs observations sur la genèse du cinéma, repose sur ce même principe de primauté. Selon eux, la double naissance d'un média se déroulerait en trois phases successives l'apparition d'une nouvelle technologie (phase 1), la mise en place d'un dispositif (phase 2), la prise de conscience d'une autonomic expressive et d'une spécificité médiatique avec tout ce que cela suppose, tant pour les socialités que pour la dimension institutionnelle du média (phase 3). François Odin observe le mêm mouvement, qualifiant la technologie émergente de « cryptomédia » (puisqu'on ne peut pas encore prévoir qu'elle va donner nais « cryptomédia » (puisqu'on ne peut pas encore prevoir qu elle va donner naissance à un média), la phase 2

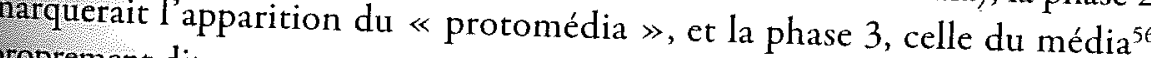
proprement dir.

La transmédialité ne fait pas que bouleverser cette chronologie, elle nie la primauté technologique. La divergence médiatique et la dynamique transmédiale qu'elle provoque tendent à montrer que le changement de technologies 'n'enédiale pas de changement de média. Et cela vaudrait aussi pour la genèse des médias.

pecularitie windows we experience a world through, the less reliant that world is on the potential for any one medium for its existence. Thus, transmediality also suggests the we see and hear continuance of a world in multiple instances and registers ; and the more that it and hear of a transmedial world, the greater is the illusion of ontological weight Primary Subereation, New, Mark J. P. Wolf, Building Imaginary Worlds. The Theory and History of teliséo Véron,

- Voir And

et Représentations, « La Croisée des Marion, « Un média nait toujours deux fois », Sociétés 
L'histoire nous enseigne [dit Jenkins] que les vieux médias ne meurent jamais - pas plus qu'ils ne disparaissent. Ce qui meurt, ce sont seulement les outils que nous utilisons pour accéder aux contenus médiatiques - les cassettes 8 pistes, les bandes Beta ${ }^{57}$.

Jenkins en vient à établir une distinction nette entre le média et ce qu'il appelle les « technologies de livraison » du contenu médiatique - les delivery technologies. « Le son enregistré est le média. Les CD, les fichiers MP3, les cassettes 8 pistes sont des technologies de livraison ${ }^{58} \gg$. Les travaux que Lars Elleström mène depuis quelques années sur la multimodalité vont dans le même sens. I subdivise le média en trois sous-catégories, le média de base, le média qualifié - auquel se rattachent les pratiques artistiques - et le média technique, concept qu'il emprunte à Friedrich Kittler et qui correspond sensiblement aux « technologies de livraison » de Jenkins.

Les médias de base et les médias qualifiés sont des catégories abstraites qui nous aident à comprendre comment des types de médias se forment par l'amalgame de qualités distinctes, alors que les médias techniques sont des dispositifs très tangibles nécessaires à la matérialisation de cas précis de ces types de médias ${ }^{59}$.

On saisit d'emblée les enjeux ontologiques et méthodologiques d'un tel changement conceptuel et les risques, qui l'accompagnent, d'un retour à une vision essentialiste des médias. Jusqu'à présent, l'identité des médias était principalement définie par la matérialité de leur médiation, mais qu'advient-il si une partie importante de celle-ci - le média technique d'Elleström ou la technologie de livraison de Jenkins - change de façon radicale ? C'est précisément à cette question que Gaudreault et Marion consacrent leur plus récent essai intitulé $L a$ fin du cinéma ? Un média en crise à l'ère du numérique. Ils se demandent si « en passant au numérique, le cinéma a simplement pris un tournant (on pourrait parler de tournant numérique) ou s'il est en train de devenir autre ${ }^{60} \gg$. Si tel est le cas, on présume qu'il faudrait l'appeler autrement. Citant, au passage Raymond

57. - [Notre traduction $] \ll[H]$ istory teaches us that old media never die - and they don't even necessarily fade away. What dies are simply the tools we use to access media content - the 8-Track, the Beta tape », Henry Jenkins, Convergence Culture. Where Old and New Media Collide, op. cit., p. 13.

58.- [Notre traduction] « Recorded sound is the medium. Cds, MP3 files, and 8-track cassettes are delivery technologie », ibid.

59. - [Notre traduction] «Basic and qualified media are abstract categories that help us understand how media types are formed by very diffe ents sorts of qualities, whereas technical media are the very tangible devices needed to materialize instances of media types Lars Elleström, « The Modalities of Media: A Model for Understanding Intermedial Relations », in Lars Elleström (dir.), op. cit., p. 12.

60.- André Gaudreault et Philippe Marion, La fin du cinéma ? Un média en crise à l'ère du num rique, coll « Cinéma/Arts visuels $\gg$ Paris, Armand Colin, 2013, p. 13.
Bellour qui affirme que le « numérique ne suffit pas à cette mort [du cinéma]; quel que soit ce qu'il perturbe à tant d'égards, il ne touche pas l'essentiel ${ }^{61} \gg$, ils s'appuient sur la généalogie du cinéma pour conclure que le cinéma n'est pas en train de mourir. Simplement, « une génération s'efface et fait place à la suivante ${ }^{62} \gg$. L'argument généalogique n'est pas sans intérêt. Au modèle de la double naissance qu'ils avaient développé en 2000, la seconde étant l'institutionnalisation du média, Gaudreault et Marion en ajoutent une troisième, celle qui se déroule actuellement, « une naissance intégrative et intermédiale, qui suppose une certaine forme de retour à la porosité, du bric-à-brac, de l'hybui suppose métissage, toutes choses qui imprégnaient la toute première naissance ${ }^{63} . \gg$ En associant la situation actuelle à une situation antéricure de l'histoire duce média deux auteurs luion actuelle à une situation antérieure de l'histoire du média, les deux auteurs lui conferent une légitimité ontologique : le numérique est médiatiquement acceptable puisque le changement qu'il produit renvoie à une situation déjà inscrite dans l'histoire du média.

Paradoxalement, puisqu'ils maintiennent l'idée d'un processus créateur qui commence par la technologie, Gaudreault et Marion accréditent le principe de rupture défendu par Jenkins et Elleström : même une pratique médiatique aussi technologisée que le cinéma peut survivre aux transformations technologiques les plus radicales.

Autant que l'éclatement du concept traditionnel de média et la fin de la primauté technologique, ce qui caractérise la pensée intermédiale actuelle - de la période postmédiatique -, c'est l'érosion des principes de déterminisme et de progrès et, plus encore, la fin de l'illusion des exclusivités médiatrices. Désormais, la question de l'identité du média devient secondaire par rapport à son action et à sa situation dans les configurations transmédiales. Non seulement savons-nous à présent que l'ensemble des caractéristiques qui permettait de définir un média est instable, sans cesse changeant, mais nous avons aussi compris qu'aucune de ces caractéristiques ne lui appartient en propre et, plus encore, qu' aucune d'elles, prise isolément, n'est décisive quant à sa survie, pas même celles qui, comme la triade eaméra-projecteur-écran, paraissent si essentielles et fondamentales. Le cas de la « présence » au théâtre, que nous abordons plus loin, confirme que la conclusion générale de Gaudrea que nous abordons plus loin, confirme que la conclusion l'ensemble Gaudreault et Marion s'applique aussi au théâtre et, sans doute, à projecteur pratiques médiatiques. Comme il existe du cinéma sans caméra, vivants. D'ou écran, il existe des spectacles théâtraux sans présence d'acteurs wants. D'où cette injonction d'Alexander R. Galloway, « pas de média, juste

61, - Raymond Billor,

coll. «Trafic », Paris, La querelle des dispositifs. Cinéma, installations, expositions, et Marion, voir Lar, P.O.L., 2012, p. 16. Les propos de Bellour sont repris par Gaudreault

2. 1 bid., p. 250.

3. 1 loid, p. p. 173 
de la médiation ${ }^{64} \gg$. Pour Galloway, en effet, l'espoir d'en arriver à une définition claire et stable du média et du dispositif de médiation est non seulement vain, c'est une chimère dont il faut se défaire en même temps qu'il faut se libérer de l'emprise de la pensée technocentriste qui l'a fait naître. La priorité accordée à la médiation marque le triomphe de la perspective performative dans l'univers des réflexions médiatiques.

Cela étant, on ne peut pas pour autant faire abstraction des questions d'ordre ontologique puisque de grands ensembles médiatiques existent, sinon dans la réalité du moins dans les discours qui en rendent compte. Revenons au cas du numérique, il aurait mis ou il aurait pu mettre en danger des éléments fondamentaux de la pratique cinématographique. Ce n'est de toute évidence pas le cas. L'objectif n'est donc plus de savoir quels sont ces éléments fondamentaux ou cet « essentiel » cinématographique, par exemple, auquel faisait allusion Bellour - puisqu' ils peuvent changer d'une conjoncture à une autre -, que de comprendre qui les définit, quand et pourquoi. L'exemple du théâtre est à cet égard particulièrement instructif et mérite, pour cela, qu'on s'y attarde.

\section{Le théâtre et la pensée intermédiale}

\section{La lente pénétration de l'intermédialité dans le champ des études théátrales}

Il aura fallu attendre près de vingt ans avant que l'intermédialité ne fasse son entrée dans le champ des études théâtrales. On peut s'étonner que le théâtre, qui a vécu de remarquables mutations grâce à l'électricité et qui connaît depuis une trentaine d'années un important renouveau, sous l'effet conjugué de l'éclatement des barrières disciplinaires traditionnelles et de l'invasion des « nouvelles technologies » (numériques) - les deux ne sont pas sans lien -, n'ait pas plus tôt attiré l'attention des chercheurs intermédiaux et n'ait pas davantage lui-même intégré à son arsenal heuristique et créatif certains concepts clés de l'approche intermédiale. Pratique fertile où se croisent et se mêlent de temps immémoriaux les arts et les technologies, où la question du dispositif est cruciale - autant que problématique -, le théâtre se trouve lié, d'une façon ou d'une autre, à tous les grands bouleversements médiatiques qui ont marqué le monde depuis près d'un siècle et demi, soit qu'il y ait contribué, soit qu'il les ait subis, soit les deux à la fois. Pourtant, Bolter et Grusin évoquent à peine le théâtre dans leur essai historique ; Jürgen E. Müller n'en traite qu'accessoirement et Walter Moser, qui a examiné les manifestations interartiales les plus diverses, n'a pas inclus le théâtre à ses observations! Ce silence s'explique sans douteen partie par l'origine disciplinaire (et les intérêts) des premiers chercheurs intermédiaux. Mais il y a davantage. En affirmant la primauté des systèmes de relations sur les objets dans la dynamique

64. $-[$ Notre traduction $] \ll[\mathrm{N}] \mathrm{ot}$ media but mediation $\gg$, Alexander R. Galloway, op. cit., p. et la genèse des médias et, surtout, en plaçant au centre de leurs préoccupations la question de la matérialité des phénomènes de médiation - ce qui implique une intervention technologique -, les premiers théoriciens de l'intermédialité n'ont rien fait pour s'attirer la sympathie d'un milieu qui, depuis près d'un siècle, échafaudait un discours identitaire fondé sur l'acteur (sa voix, son corps) et son rapport « immédiat », entendons par là non technologiquement médiatisé, au spectateur : en un mot, sur la présence. Le paradoxe de cette situation est que le concept de présence qui a causé une telle « résistance » à la pénétration des idées intermédiales est lui-même le produit de la dynamique intermédiale. Il fallait, pour s'en rendre compte, adopter une approche intermédiale !

\section{Approche intermédiale de la présence au théatre}

La présence est aujourd'hui si liée à l'épistémè thêâtrale qu'on a peine à les distinguer l'une de l'autre. Soyons clairs, questionner le concept de présence ne remet pas en cause le fait que le dispositif théâtral, comme celui d'autre pratiques (la danse, le concert), ait généralement et historiquement reposé sur la présence simultanée, en un même lieu physique, d'un acteur er d'un spectateur vivants. Mais cela ne justifie pas d'ériger la présence en caractéristique nécessaire et irréductible - « essentielle 》 - de la médialité théâtrale. C'est pourtant ce qui s'est produit, la présence devenant l'élément clé de la stratégie d'affirmation identitaire du théâtre dans le contexte de la vaste reconfiguration médiatique provoquée par l'avènement de l'électricité. Ayant ceci en tête, on ne s'étonnetique pas que le concept de présence, tel que nous le comprenons aujourd'hui, soit né en même temps et à cause des technologies de reproductions du son et de l'ime ne qui ont donné naissance aux grands médias de la première moitié du XX $\mathrm{XX}^{c}$ siècle puisque le théâtre a dû réagir à la concurrence en memière moitié du XX' $\mathrm{XX}^{\mathrm{c}}$ sècle, distinguait. On trouve reagir à la concurrence en mettant de l'avant ce qui l'en cette déclaration reuve une trace annonciatrice de cette stratégie distinctive dans liste du New Yon retentissante qu'a faite l'Américain Daniel Frohman à ụn journadébut du XX' serk Times. Producteur de théâtre très influent, Frohman était, au opinions a siecle, l'une des personnalités les plus en vue de Broadway et ses années, divaient d'autant plus de poids qu'il avait aussi été, pendant quelques années, directeur de la Famous Players Film.

Le cinéma estémaintenant [en 1915] à son zénith.

$[\ldots .$.

$[\mathrm{II}] \mathrm{e}$ probl

II pourra y arriver cinéma n'est pas d'augmenter son public mais de le conserver

La synchronisation perfectionnant encore davantage son art déjà merveilleux.

matière explicative du son er du mouvement à l'écran, un procédé par lequel la

éviterait d'interrve maintenant imprimée sur le film pourrait être dite, ce qui

stéreoscopique aur in le film, la reproduction de la couleur et l'ajout d'un effet gens travaillen aux images sont des améliorations sur lesquelles beaucoup de 6ens travaillent. Mais l'image animée, élevée au énième degré des beaucoup de 
pourra jamais complètement supplanter le drame parlé. En voici la raison : il se peur bien qu'on réussisse à synchroniser le son et le mouvement et que les se peur bien qu'on reusisse à synchroniser le son et le mouvement et que les visages à l'écran donnent l'impression qu' ils parlent vraiment, mais cette qualité humaine qu'on appelle la personnalité ne pourra jamais être traduite par une lentille er transmise aux spectateurs par le médium de l'écran. Seule la présence d' l'acteur vivant peut communiquer le magnétisme de l'acreur au public ${ }^{65}$.

Cent ans plus tard, si certains aspects de la déclaration de Frohman font sourire, d'autres étonnent par leur actualité. Le recours à l'argument de la présence physique (plus loin dans le même article, il évoque une présence « en chair et en os » - « offlesh and blood ») est de ceux-là. À l'époque, l'argument visait les spectateurs de théâtre qui commençaient à le déserter en grand nombre. Frohman voulait les convaincre de rester fidèles au théâtre en invoquant la supériorité ontologique de celui-ci sur les autres pratiques qui le concurrençaient. De toute évidence, la stratégie n'a pas réussi. N'empêche, l'argument a été repris et se trouve à la base de deux théories capitales qui ont marqué le discours théâtral et le discours médiarique du XXe siècle : la théorie de l'aura - et donc la question de l'impact de la reproduction technique sur les pratiques artistiques - qu'a explorée Walter l' définie Henri Gouhier dans Benjamin et celle de la présence scénique telle que l'a définie Henri Gouhier dans son livre, devenu livre-culte, L'essence $d u$ théatre, dont la première parution date de $1936^{66}$. Qu'est-ce que le théâtre, demande Henri Gouhier ? C'est « l'art de représenter $\gg$. Gouhier n' inventait rien, se contentant de dire autrement la vieille théorie mimétique héritée d'Aristote et remise à jour, depuis lors, à chaque grande théorie mimetique heritée. Mais qu'est-ce que représenter précisément? « [C]'est rendre présent par des présences ${ }^{67} \gg$. L'essai de Gouhier est une longue variation sur ce thème unique : le théâtre est l'art de la présence. Ces deux théories, celle de l'aura qui s'estompe sous le coup de la reproductibilité technique et celle de la présence, que peut seule créer la coprésence physique - vivante - de l'acteur

65. [Notre traduction] « The moving picture is now at the zenith of its power. [...] [T] he pro blem of he cinema is not to increase its public, but to hold it. This it will do by perfec blem of the cynchronization of sound with motion on ting still further its already the screen, a device by which the explanatory matter now printed on the film will not be interrupted, the reproduction of color, and the giving of a stereoscopic quality to the pic tures are improvements on which many men are at work. But the moving picture raised to the nth degree of perfection can never completely supplant the spoken drama. This is because, while sound and motion may be synchronized eventually so the figures of the screen will give every appearance of speaking, that human quality we call personality car screen willgive evry appeat never be translated by the lens and transmitted to the audience through the medium of screen. Only the presence of the living player can communicate the player's magnetism to the audience », Daniel Frohamn, cité dans l'article The Movie Here to Make the Spoke Drama Behave », The New York Times, 9 mai 1915, p. X6.

66. Il sera régulìrement réédité depuis, dont la dernière fois en 2002.

67- Henri Gouhier L'essence du théátre, coll. «Bibliothèque d'histoire de la philosophie 》 Paris, Vrin, 2002 [1936], p. 16. et du spectateur, sont exactement contemporaines de deux percées médiatiques majeures, la radio et le cinéma parlant, ce qui n'est évidemment pas un hasard.

Pour des raisons qui restent à élucider, le discours théâtral sur la présence s'est appuyé sur le vieux sentiment anti-mécaniste, né au XIX ${ }^{\mathrm{e}}$ siècle, dont Frohman lui-même s'était fait l'écho et qui perpétuait, sous une forme renouvelée, la dox religieuse de la supériorité du divin (le naturel) sur l'humain (l'artificiel). Le concept de présence s'est ainsi chargé d'une forte connotation anti-technologique et la scène théâtrale a progressivement pris la valeur emblématique de l'ultime refuge de la communication humaine « vraie », directe, « naturelle $»$ dan un monde nouveau, inquiétant, marqué par l'artifice, celui de la reproduction technologique. La démarche reposait sur un préjugé tenace : rien ne surpasse en efficacité et en précision la communication de bouche à oreille entre deux personnes présentes « en chair et en os », ce que la réalité dément pourtant quotidiennement. Or, à la même époque, la radio proposait un autre concept de présence fondé sur l'intimité et l'individualité - de la personne qui parle au micro à celle qui l'écoute dans son salon -, qu'elle opposait à l'indistinction du spectateur de théâtre, noyé dans une masse, rendu anonyme. Ceci illustre bien l'élasticité du concept. Petit à petit, donc, la scène théâtrale est devenue lantithèse du bric-à-brac technologique des studios de disque, de radio ou de cinéma, créateurs de factice, et le théâtre s'est affirmé comme le champion « de la culture pure et authentique dans un monde de vacuité mass-médiatique et télévisuelle ${ }^{68} 》$, ainsi que le souligne avec ironie Peter Boenisch. De là résulta l'idée que la présence est le contraire du médiatisé, la première étant vraie, le second étant faux. Et cette idée s'imposa durablement. Peggy Phelan la défendait encore avec virulence dans son essai Unmarked. The Politics of Performance, publié en 1993, alors que les technologies médiatiques numériques entraient massivement dans la pratique, menaçant, à ses yeux, $l^{\prime} \ll$ intégrité » de la scène traditionnelle, en profanant le « refuge » par l'introduction de projections vidéo ou de sons reproduits ou, pire encore, en saisissant l'éphémère de la scène avec une caméra de façon à pouvoir la saisir et la reproduire.

Seule la vie est au présent. La représentation [théâtrale] ne peut pas être sauvegardée, enregistrée, documentée ou entrer de quelque façon que ce soit dans la circulation de représentations de représentations; si elle fait cela, elle devient
autre chose. : $[+.,+1$.

68. [Notre tra

daftness 》 Petion] « of pure and authentic culture in a world of mass-media and television Performance », in Freda Chapple et Chiel Kattenthetic Act: Theatre, Media, Intermedial 
prévisible. Son déclin était irréversible. La pénétration massive des technologies Plus une représentation [théâtrale] tente d'entrer dans l'économie de la reproduction, plus elle trahit et affaiblit les espoirs de sa propre ontologie ${ }^{69}$

Cet argument, qu'ont contredit les théories de Bolter et Grusin sur l'hyperCe Kartenbelt sur l'hypermédialité ou, encore, de Wolf sur le poids onéd dable effer mobilisateur ce qui explique la violence du débat qu'il a déclenché «The Phelan-Auslander Debate », qui a déchiré le champ anglophone des études théâtrales pendant près de dix ans, a eu pour principal mérite de sortir les Performance Studies, fortement marquées dès leur origine par la pensée phénoénologue de la torpeur où les maintenait le discours essentialiste ambiant. Ce débat a connu son paroxysme en 1999 avec la publication de Liveness. Performance in adiatized Culture de Philip Auslander. Cet ouvrage, qui a eu l'effet d'une bombe, parut quelques mois à peine avant l'autre essai phare de cette période, celui de Bolter et Grusin sur la remédiation dont il partageait les valeurs intermédiales. Auslander y défendait deux idées majeures diamétralement opposées celles de Phelan. D'une part, la présence - the liveness ${ }^{70}$ - n'est pas l'apanage lusif du théâtre ; de nombreuses pratiques médiatiques, artistiques ou non - comme les cours de justice -, intègrent la présence à leur dispositif. Il s'agit là d'une première attaque frontale contre l'unicité prétendue, presque sacralisée de la scène théâtrale (comme 《ultime refuge »). D'autre part, et ceci est beaucoup lus important la présence n'est pas, n'a jamais été le contraire du médiatisé et elle n'est pas incompatible avec le médiatisé (comme l'illustrent la radio et, plus 列 les plus technologiques et la scène en apparence la moins technologisée, comme celle de Jerzy Grotowski ou de Peter Brook, reste un dispositif médiatique et, tout dénudé qu'il soit, le corps de l'acteur communiant avec son public en fait partie. La présence, au théâtre, est toujours le produit d'une médiation, un effet.

L'électrochoc causé par Auslander n'a eu pour effet immédiat que de Lencore davantage une pensée essentialiste en plein désarroi. C'étai

docirculion of representations of representa documented, or other than performance. [...] To the degre tions: once it does so, it becomes som lessen that performance attempts to enter the economy of inmarked. The Politics of Performance the promise of its own ontology 》, Peggy Phelan, Unmarked The Polites of Perfom Londres/New York, Routledge, 1993, p. 146.

70.- Bien que les deux termes ne soient pas absolument équivalents, ils recourrent à peu pres même champ sémantique.

71.- Voir en particulier le texte de Jay David Bolter, Blair Mal Media », in Jürgen E. Müller (dir. Schweitzer, «Benjamin's Crisis of Aura and Digital Media », in in der Diskussion Media Encounters and Media Theories, coll.

numériques sur scène et dans les processus de création du théâtre, qui s'accélérait durant cette période, loin de « trahir les espoirs de sa propre ontologie », faisait la démonstration du caractère spécieux de l'appel à l'« authenticité » et donnait raison à Auslander.

Le fait que la première percée marquante de la pensée intermédiale dans le champ des études théâtrales survienne en 2006, alors que le débat PhelanAuslander s'estompe, n'est pas une coüncidence. Intermediality in Theatre and Performance, que co-signaient quinze chercheurs membres de la Fédération internationale pour la recherche théâtrale (FIRT) sous la direction de Freda Chapple et de Chiel Kattenbelt, ouvrait une ère nouvelle autant dans le champ des études théâtrales que dans celui de l'intermédialité. Mais il aura fallu, pour cela, venir a bout de l'hégémonie du discours essentialiste sur le monde du théâtre et voir s'effondrer le mythe de la «pure » présence et avec lui une vision idéale du théâtre. Pour Roger Copeland,

l'idée que la présence soit en soi et de soi une vertu, une source qui confere automatiquement au théâtre, et sans aucun mérite de sa part, une supériorité morale sur le film et la télévision est une pure sentimentalité bourgeoise ${ }^{72}$.

Depuis, des pans entiers de la pratique théâtrale et des questions majeures mais longtemps occultées comme « l'autre coprésence » (de l'humain et de la technologie), le son, la magie, le virtuel s'ouvrent à la recherche et à la réflexion théâtrales; des idées de fond comme les concepts de représentation, de culture participative, de virage affectif - theatre feelings - ; la distinction entre théâtralité et performativité, entre théâtre et performance, tout cela fait l'objet de réexamens attentifs et de projections stimulantes, telle cette idée d'un « théâtre postmédiatique » qu'évoque Simon Hagemann ${ }^{73}$ et qui pourrait résulter de l'effet combiné des avancées de la pensée et des pratiques intermédiales et théâtrales. Au demeurant, on ne peut nier, à l'instar d'Amy Petersen Jensen, qu'une part de nostalgie subsiste et subsistera ${ }^{74}$, mais s'il y a nostalgie, c'est moins pour cette figure de l'origine que l'intermédiabilité a, dès le début, liquidée que pour tout un

72.-[Notre traduction] « Furthermore, the idea that theatre's liveness is - in and of itself virtue, a source of automatic, unearned moral superiority to film and television, is sheer bourgeois sentimentality », Ror Copeland Drama Review, vol. 34, $\mathrm{n}^{\circ} 4$, hiver 1990 eland, « The Presence of Mediation », TDR: The -Simon Hagem vol. 34, n' 4, hiver 1990, p. 42

contemporaines coll Penser les médias au théatre. Des avant-gardes historiques aux scènes 2013. p. 250. coll. « Ouverture philosophique. Série Arts vivants », Paris, L'Harmattan, Any $\mathrm{P}$ Since 1970, Jeffersen, Theatre in a Media Culture. Production, Performance and Perception (Caroline du Nord)/Londres, McFarland, 2007, p. 7. 
Galloway, Alexander R., The Interface Effect, Cambridge, Polity, 2013 [2012], 170 p.

pan de la dynamique intermédiale occulté par des discours identitaires devenus aujourd'hui impraticables et indéfendables.

S'il y a ici nostalgie dans la pensée intermédiale actuelle, c'est de toure évidence loujours-déjà-nouveau derridien.

«The Movie Here to Make the Spoken Drama Behave », The New York Times, 9 mai 1915, p. X6.

ACLAND, Charles R. (dir.), Residual Media, Londres/Minneapolis, University of Minnesota Press, 2007, $401 \mathrm{p}$ thèque », trad. de l'italien de Martin Rueff, Paris, Payot Rivages, 2007, $50 \mathrm{p}$

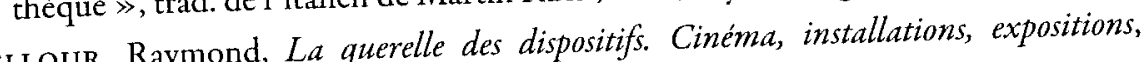
coll. «Trafic », Paris, P.O.L., 2012, 573 p.

Féréric et Catherine Bertho-Lavenir, Histoire des médias. De Diderot a Internet, coll. «U $\mathrm{U}$, Paris, Armand Colin, 1996,351 p

Boenisch, Peter, « Aesthetic Art to Aistheric Act: Theatre, Media, Intermedia Performance », in Freda Chapple et Chiel Kattenbelt (dir.), Intermediality Perfore Rodopi, p. 103-116.

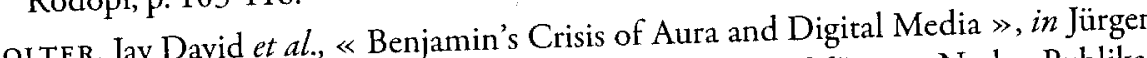
Medis E. Müller (dir.), Media Encounters and Media Theories, Munster, Nod

tionen, coll. «Film und Medien in der Diskussion », Understanding New Media Bolter, Jay David et Richard Grusin, Remediation. 295 p.

Cambridge (Massachusetts), MIT Press, 2000 [1999], 295 . Chapple, Freda et Chiel Kattenbelt, « Key Issues in Intermedialiy and Perfor mance », in Freda Chapple et Chiel KatTenbelt (dir.), Intermediality in Theatre and Performance, coll. « Themes in Theatre », Amsterdam/New York, Rodopi, p. 11-25.

Cüver, Clats « Intermediality and Interarts Studies », in Jens Arvidson, Mikael Askan ContemASKANDER, Jorgen BRUHN et Heidrun FU TRed (dia Studies Press, 2007, p. 19-37. porary Positions in Intermediality, Lund, Intermedia Studies Press, $2007, \mathrm{p} .1937$.

Copeland, Roger, «The Presence of Mediation », TDR: The Drama Review, vol. 34 $n^{\circ} 4$ hiver 1990, p. 28-44

Davis, Tracy C et Thomas Postuewait (dir.), Theatricality, coll. « Theatre and Performance Theory », Cambridge, Cambridge University Press, 2003, $243 \mathrm{p}$

Donguy, Jacques et Charles Dreyfus, Dick Higgins, 1938-1998. Intermédia, Québec Éditions Intervention, $1999,20 \mathrm{p}$

Elleström, Lars (dir.), Media Border, Multimodality and Intermediality, New York Palgrave Macmillan, 2010,270 p.

Entrée « Technology » du dictionnaire Meriamm-Webster, < http://www.merria webster.com/dictionary/technology > (consultation le 10 juillet 2014).
Gaudreault, André et Philippe Marion, «Un média naît toujours deux fois », Sociétés et Représentations, «La Croisée des médias », $\mathrm{n}^{\circ}$ 9, avril 2000, p. 21-36. La fin du cinéma? Un média en crise à l'ère du numérique, coll. «Cinémal Arts visuels », Paris, Armand Colin, 2013, 275 p.

Gitelman, Lisa, Always Already New. Media, History and the Data of Culture, Cambridge (Massachusetts)/Londres, MIT Press, 2006, $205 \mathrm{p}$

Gounier, Henri, L'essence du théatre, coll. « Bibliothèque d'histoire de la philosophie », Paris, Vrin, 2002 [1936], 206 p.

Hagemann, Simon, Penser les médias au théâtre. Des avant-gardes historiques aux scènes contemporaines, coll. « Ouverture philosophique. Série Arts vivants », Paris, L'Harmattan, 2013, $262 \mathrm{p}$.

HigGins, Dick, Intermedia (rédigé en 1966 et publié en 1967 dans la Something Else Newsletter), disponible au < hrtp://www.artpool.hu/Fluxus/Higgins/intermedia2. html > (consultation le 10 juillet 2014)

Jenkins, Henry, « Convergence and Divergence: Two parts of the same Process », Confession of an Aca-Fan. The Official Wenblog of Henry Jenkins, 29 juin 2006, $<$ htrp://henryjenkins.org/2006/06/convergence and divergence two.hrml > consultation le 12 mars 2004)

Convergence Culture. When Old and New Media Collide, New York, New York University Press, 2008 [2006], 336 p.

Jensen, Amy Petersen, Theatre in a Media Culture. Production, Performance and Perception Since 1970, Jefferson (Caroline du Nord)/Londres, McFarland, 2007. $221 \mathrm{p}$.

Kattenbelt, Chiel, « Theatre as the Art of the Performer and the Stage of Intermediality », in Freda Chapple et Chiel Kattenbelt (dir.), Intermediality in Theatre and Performance, coll. « Themes in Theatre », Amsterdam/New York, Rodopi, p. 29-39.

Krrcknove (De), Derrick, « A Theory of Greek Tragedy 》, SubStance, vol. $4, n^{\circ} 9$, iss. 29,1980, p. $23-36$

Lamham, Richard, The Electronic Word. Democracy, Technology, and the Arts, Chicago, University of Chicago Press, 1993, 285 p.

LARruE, Jean-Marc, « Sound Reproduction Technique in Theatre: A Case of Mediatic Resistance », in Lynne Kendrick et David Roesner (dir.), Theatre Noise. The Sound of Performance, Cambridge, Cambridge Scholars, 2011, p. 14-22.

Arrue, Jean-Marc et Giusy Pisano (dir.), Les archives de la mise en scène. Hypermédialites du théatre, Lille, Presses Universitaires du Septentrion, 2014, $422 \mathrm{p}$.

nication, Carolyn, When Old Technologies Were New. Thinking About Electric Commu269 $269 \mathrm{p}$

Melunan, Marshall Pour comprendre les médias. Les prolongements technologiques de 390 p. 


\section{La performance médiatisée, un défi aux études théâtrales ${ }^{1}$}

Ralf Remshardt

Phelan, Peggy, Unmarked. The Politics of Performance, Londres/New York, Rourledge, $1993,207 \mathrm{p}$.

Rancière, Jacques, « Ce que "medium" peut vouloir dire : l'exemple de la photographie », Revue Appareil, $\mathrm{n}^{\circ}$ 1, 17 février 2008, < http://appareil.revues.org/135> (consultation le 30 juin 2014)

SALTER, Chris, Entangled. Technology and the Transformation of Performance, Cambridge (Massachusetts), MIT Press, 2010, $460 \mathrm{p}$.

(dir.),

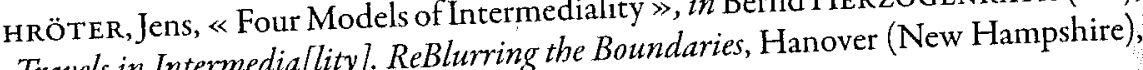
Travels in Intermediallity]. ReBlurring the Bo

Dartmouth College Press, 2012, p. 15-36.

Sibony, Daniel, Entre-deux. L'origine en partage, coll. « La couleur des idees », Paris, Éditions du Seuil, 1991, 398 p.

SMITH, Jacob, Vocal Tracks. Performance and Sound Media, Berkeley, University of California Press, 2008, $294 \mathrm{p}$

Calo, Arcologie, la ville à l'image de l'homme, Marseille, Parenthèses, 1980 $132 \mathrm{p}$.

She Meaning of a Format, coll. « Sign, Storage, Tran mission $\gg$ Durham/Londres, Duke University Press, 2012, 341 p.

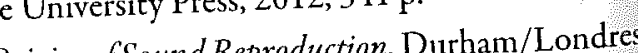
The Audible Past. Cultural Origins of Sound Reproduction, Durham/Londres Duke University Press, 2003, $450 \mathrm{p}$.

Véron, Éliséo, « De l'image sémiologique aux discursivités. Le temps d'une photo Hermés, «Espaces publics en images », $\mathrm{n}^{\circ \mathrm{s}} 13-14,1994, \mathrm{p} .45-64$.

Wor Mark J. P., Building Imaginary Worlds. The Theory and History of Subcreation New York, Routledge, 2012, $394 \mathrm{p}$.

Z Seing by Technical Means, Cambridge (Massachusetts)/Londres, MIT Press, 2006 $375 \mathrm{p}$. 